\title{
Disparity in HIV Service Interruption in the Outbreak of COVID-19 in South Carolina
}

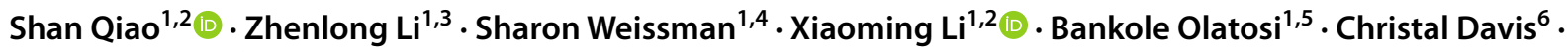 \\ Ali B. Mansaray ${ }^{6}$
}

Published online: 27 August 2020

○) Springer Science+Business Media, LLC, part of Springer Nature 2020

\begin{abstract}
To examine HIV service interruptions during the COIVD-19 outbreak in South Carolina (SC) and identify geospatial and socioeconomic correlates of such interruptions, we collected qualitative, geospatial, and quantitative data from 27 Ryan White HIV clinics in SC in March, 2020. HIV service interruptions were categorized (none, minimal, partial, and complete interruption) and analyzed for geospatial heterogeneity. Nearly $56 \%$ of the HIV clinics were partially interrupted and $26 \%$ were completely closed. Geospatial heterogeneity of service interruption existed but did not exactly overlap with the geospatial pattern of COVID-19 outbreak. The percentage of uninsured in the service catchment areas was significantly correlated with HIV service interruption $(\mathrm{F}=3.987, \mathrm{P}=.02)$. This mixed-method study demonstrated the disparity of HIV service interruptions in the COVID-19 in SC and suggested a contribution of existing socioeconomic gaps to this disparity. These findings may inform the resources allocation and future strategies to respond to public health emergencies.
\end{abstract}

Keywords COVID-19 $\cdot$ HIV service $\cdot$ South Carolina $\cdot$ Health disparity $\cdot$ Geospatial data

\section{Introduction}

The outbreak of coronavirus disease 2019 (COVID-19), caused by the severe acute respiratory syndrome coronavirus (SARS-CoV-2), is a rapidly evolving global public health crisis [1]. On March 11, 2020, the World Health

Shan Qiao

shanqiao@mailbox.sc.edu

1 The South Carolina SmartState Center of Healthcare Quality, Arnold School of Public Health, University of South Carolina, Columbia, SC, USA

2 Department of Health Promotion Education and Behavior, Arnold School of Public Health, University of South Carolina, Columbia, SC, USA

3 Department of Geography, University of South Carolina, Columbia, SC, USA

4 Department of Internal Medicine, School of Medicine, University of South Carolina, Columbia, SC, USA

5 Department of Health Services Policy and Management, Arnold School of Public Health, University of South Carolina, Columbia, SC, USA

6 South Carolina Department of Health and Environment Control, Columbia, SC, USA
Organization characterized COVID-19 as a pandemic [2]. As of August 5, 2020, infection cases exceeded 18,614,177 across 216 countries and areas, with over 702,642 deaths [3]. These numbers continue to rise daily as the virus continues to spread globally.

People living with HIV (PLWH) have been recognized as a medically and socially vulnerable population during the COVID-19 pandemic [4]. HIV-related services have been unavoidably interrupted and impacted. On March 18, 2020, the CDC posted specific guideline to address PLWH's concerns and questions related to their COVID-19 risk and prevention. On March 20, 2020, the NIH Office of AIDS Research also provided interim guideline related to COVID19 and PLWH. However, there have been many challenges in the implementation of these guidelines, particularly in the areas of healthcare delivery and PLWH's linkage to care and retention in care $[5,6]$.

During the unprecedented COVID-19 outbreak, many health facilities providing HIV prevention, treatment and care services had to change their schedules and operation modalities to adapt to the challenges imposed by COVID-19 and its prevention and control measures (social distancing, travel restrictions, stay at home orders, etc.). Routine, nonurgent or elective health care visits and procedures were 
cancelled or delayed. Supportive services such as face-toface counselling, housing services and outreach services were temporarily suspended. In addition, patients avoided accessing health care, even for urgent concerns, due to fear of COVID-19 exposure [7, 8]. In order to improve healthcare delivery and provide sustainable quality care to PLWH during the COVID-19 pandemic and future pandemics, we need a better understanding of the nature and scope of HIV treatment service interruption and their correlates.

Existing literature suggests a health disparity in COVID19 prevalence and related clinical outcomes (e.g., complications, mortality) in the United States. For example, one recent spatial analysis on COVID-19 epidemic reported an uneven impact on incidence and mortality of COVID-19 in the United States [9]. The study showed positive correlations of COVID-19 incidence and mortality rates with socioeconomic factors [9]. However, no empirical studies have examined if there is a spatial disparity in the HIV service interruption and if existing social determinants of health influence the pattern of HIV service interruption, particularly in Southern States such as South Carolina.

South Carolina (SC) has been heavily hit by the COVID19. The first case of COVID-19 was reported in SC on March 6, 2020 [10]. On April 2, 2020, SC Department of Health and Environmental Control (DHEC) announced that the virus had spread to all 46 counties in the state [11]. As of August 5, 2020, COVID-19 cases exceeded 94,831 with over 1819 deaths in SC [10].

Therefore, using mixed-method data, the current study aims to (1) examine the interruption of HIV service delivery in SC during COVID-19 outbreak; (2) demonstrate the pattern of this service interruption by geolocation; and (3) identify any existing health disparity factors (e.g., socioeconomic status) that were associated with the HIV service interruption.

\section{Methods}

\section{Study Setting}

$\mathrm{SC}$ is one of the seven states targeted by the "Ending the HIV Epidemic (EtHE): A Plan for America" [12] campaign due to its high rural HIV burden [12]. With an HIV infection rate of 15.8 per 100,000 people, SC ranked 11th among all 50 states in 2018, with an estimate of 20,166 PLWH [13]. African Americans and men who have sex with men (MSM) are disproportionately affected by HIV/AIDS in SC [13]. By 2018, there were 27 clinics in SC funded by Ryan White HIV/AIDS Program [14]. The Ryan White HIV/AIDS Programs are federally funded programs to provide comprehensive HIV primary medical care, essential support services, and medications for low-income PLWH who are uninsured and underserved [15]. According to the program profile, it was estimated that 11,369 (56\%) PLWH were served by the Ryan White clinics in SC in 2018 [16].

\section{Data Collection}

Qualitative information was extracted from the program process reports of 27 Ryan White HIV clinics (grantees of Ryan White HIV/AIDS programs) across 46 counties in SC in March, 2020. These reports were submitted to the SC DHEC based on the clinics' status of operation and HIV-related service delivery during the COVID-19 outbreak.

Geolocations and zip codes of the HIV clinics were retrieved for geospatial analysis. Based on the information provided by the HIV clinics, we identified the service catchment areas of the clinics (i.e., counties these HIV clinics served). We then extracted data from various sources for each county's relevant characteristics, such as number of PLWH per 100,000 in 2018 (via AIDSVu, https://aidsv u.org), number of COVID-19 cases per 100,000 as of June 13, 2020 (via SC DHEC, https://scdhec.gov), and socioeconomic status (SES) indicators in 2016 (via US Census Bureau, https://census.gov/). The SES indicators included poverty (percent of population living below federal poverty line) [17], high school education (percent of population with a high school degree or equivalent) [18], median household income [17], Gini Coefficient (a measure of income inequality with 0 reflecting complete equality and 1 reflecting complete inequality) [19], and percent of population without health insurance [20]. The current study was approved by the Institutional Research Board at University of South Carolina.

\section{Categorization of Service Interruption}

Based on qualitative data regarding the status of operation at HIV clinics, a medical expert with over 20 years experiences in both HIV-related research and clinic care initially categorized HIV service interruption during the COVID-19 outbreak into four types: complete interruption, partial interruption, minimal interruption, and no interruption. Specifically, there were four criteria for assessing the HIV service interruption including clinic operation hours (e.g., whether clinics are open for most of weekdays), HIV service coverage (e.g., whether clinics maintain most of existing services), telehealth use (e.g., whether clinics use telehealth in service delivery), and healthcare providers' availability (e.g., whether most of healthcare providers, especially medical case managers can still work in their original capacity). The clinics that failed in meeting the four criteria were considered as "complete interruption", failed in any two or three as "partial interruption", failed in any one of the criteria as 
"minimal interruption", and those met all the four criteria as "no interruption". Another team member reviewed the data and confirmed the initial categorization using the same criteria.

\section{Data Analysis}

We analyzed the qualitative data based on grounded theory [21]. Five themes occurred regarding HIV service interruption, including operation days and time, protection measures, service coverage, the use of telehealth and mobile applications (apps), and healthcare provider availability.

We then mapped the pattern of HIV service interruptions using the Geographic Information System (GIS) to indicate geospatial distribution.

For quantitative data, we developed the county-level indicators (e.g., SES, COVID-19 cases, and HIV cases) for each HIV clinic based on its service catchment area. Specifically, each of the five SES indicators, number of COVID-19 cases per 100,000 people, and number of PLWH per 100,000 people were averaged among counties that each clinic served. It should be noted that there may be overlap among some clinics in catchment area with some counties being served by more than one clinic. In such cases, indicators of those counties were used by each serving clinic. ANOVA was used to examine the associations between these countylevel indicators and types of service interruption. One HIV clinic that provided state-wide HIV service was removed from the county-level geospatial analysis and quantitative analysis since its service catchment area was not limited to any specific county.

\section{Results}

\section{HIV Service Interruption in SC}

Among the 27 HIV clinics in the current study, two clinics (7.4\%) were assessed as "no interruption", three (11.1\%) as "minimal interruption", 15 clinics $(55.6 \%)$ as "partial interruption", and seven clinics (25.9\%) as "complete interruption". The qualitative information regarding HIV service interruption during the outbreak of COVID-19 covered five domains (See sample quotes in Table 1). In terms of operation time, only one clinic had their main site open for normal hours. Most of HIV clinics reduced their office hours, limited office visits, and/or limited face-to-face appointments. Seven clinics were closed with only staff checking emails daily. Clinics adapted various strategies in adjusting operation hours. Some canceled evening hours. Some adjusted their schedule, providing different types of services at different dates and hours.
All the clinics adapted specific measures to protect patients, visitors, healthcare providers, and staff from COVID-19 infection. Common strategies included requiring temperature and symptom screening for all visitors entering into the building, asking all the people in the offices and building to wear face masks, and taking precaution to maintain social distancing in offices. One clinic mentioned that they provided COVID-19 testing at their sites. One clinic highlighted that their patients were screened and triaged prior to entering into clinic. Another clinic reported that they had sufficient stock to provide masks to both clinic staff and visiting patients.

Due to the closure and reduced operation hours, HIVrelated services were interrupted at many clinics in terms of scope and delivery modality. Two HIV clinics reported that they had to discontinue home visits and support groups. Several clinics suspended walk-in services. One clinic mentioned that their dental offices were only seeing patients for emergency visits. Prevention services were available by appointment only and HIV testing services were provided at alternative sites. However, most of clinics still accepted new referrals for HIV treatment and primary care, and they also delivered medical care, case management services, pharmacy services and lab services over telephone and/or in the offices when needed. One clinic reported that they also provided curbside/drive through COVID-19 testing on-site and partnering with SC DHEC to do COVID-19 testing in the community. Two clinics also assisted patients with medication pick-up/delivery, food cards, personal pantry and housing services/support as needed.

Telehealth and mobile apps were widely used in HIV clinics for making appointments and delivering HIV prevention, treatment, and care services. One clinic also introduced an on-line request system for rent, food, and clothing assistance. For the clinics where regular operation hours remained unchanged, telehealth was also provided as an option for patients. One clinic reported, "We have virtual visit capabilities for all patients with regular follow-up appointments if patients have computer and internet access at home. For Ryan White patients, medical case managers (MCMs) will bring laptop and hot spot to the patient's home, get them online with provider and step out of the room".

During the COVID-19 outbreak, most healthcare providers and staff had flexible working schedules. Policy regarding remote working and return-to-work varied across clinics and also changed along with the rapidly evolving pandemic. Most of clinics reported that the providers and staff were working from home using Virtual Private Network (VPN) for access to Provide Enterprise (Ryan White data reporting 
Table 1 Summary of HIV service interruption by domains

Domains $\quad$ Sample quotes from the clinical reports

Operation (operation days and times) "Our main site is operating on normal business hours (satellite sites are currently closed)."

"Testing by appointment on Tuesday $9 a-12 p \& 1 p-4 p$. In clinic provider appointments on Monday and Thursday 9a-3p, Telehealth appointments Tuesday, Wednesday and Friday from 9a-3 pm. Clinic staff are available to answer phones every day from 8:30-5:30. Pharmacy is open Monday, Tuesday and Thursday 8:30-5:30, via phone Wednesday and Friday."

"Restricting face-to-face visits, restricting patients only to building."

"Closed for 14 days; $\mathrm{M}$ is checking and responding to emails remotely"

"Currently, offices are operating on normal hours with some limited closures for evening hours based on staffing per day."

"As of May 18, 2020 New Horizon Family Health Services will begin seeing patients in the office setting with continued reduced hours of operation."

"Office is closed. Working remotely."

"Staff in office for services Monday, Wednesday, Friday 9a-1p. All staff available 8:30-5 by telephone. Using telehealth for most medical appointments, otherwise Tuesday 3P-7P and Thursday 10a-2p. Testing on Thursday 3-7PM and 1st and 3rd Saturday 10-2 PM. Limited in office visits. ...Telephone messages checked 4 times/day. On call staff available 24/7."

"Limited office appointments, all MCM's are working in the office full-time as of Monday, June 1, 2020."

Protection measures (for healthcare workers, staff and visitors)

Service provided
"[***] has resume our regularly work schedule with taking precautions and practicing social distances in the office."

"All staff are screened daily when entering the building and wearing masks. Patients entering the building are screened and also wearing masks."

"All patients are required to wear a mask when entering the facility."

"Patients/clients/employees are required to wear masks in all locations \& facilities. We have sufficient stock to provide masks to those without."

"Patients are COVID-19 screened \& triaged prior to entrance into clinic."

"We request patients to practice social distancing, self-social isolation, and wear a mask while in clinic for appointments."

"We are providing curbside/drive thru COVID-19 testing on-site and partnering with DHEC to test within the community. We continue to provide medical care, behavioral health and dental services using telehealth and in person visits. MCMs are providing case management services over the phone and in the office when needed. We are assisting with food cards, personal pantry and housing services/support as needed. Prevention is providing [HIV] testing services on-site"

"We have also resume(d) testing and have giving appointments if a person calls prior but will not turn away walk-ins.... We are accepting new referral for HIV treatment and primary care."

"Prevention services continue to be available by appt only; however, they began providing testing services at alternative sites on June 1."

"Patients who must have face-to-face appointment with provider we are still open."

"Are limiting patients coming to the office and exploring other options such as the Healow app and telehealth visits. Our dental offices are only seeing patients for emergency visits at this time."

"Newly diagnosed patients are being seen in clinic. Patients requiring sick visits are also being seen."

"Appointments are encouraged, however walk in care will be available on a limited basis. All pharmacy services also available. Ryan White Program is fully staffed and routine clinic hours are in place. Patients can be seen with an appointment for ID as well as Lab services."

"Introduced online request systems for rent, food, and clothing assistance; online scheduling for tax assistance; no change to HIV connected services at this point other than requesting partners not drop off applications in person."

"Medications are being delivered as needed. Any of the MCM's can be reached via telephone and email."

"We are still accepting new referrals and will maintain adequate staffing. We have discontinued support groups and home visits but all other services remain the same."

"Staff is in the office conducting client appointments by telephone. We are accepting referrals and scheduling/completing intakes \& appts for emergency financial assistance and providing supportive services as needed (e.g. medication pick up/delivery, food banks). We've temporarily suspending home visits \& support groups." 
Table 1 (continued)

\begin{tabular}{|c|c|}
\hline Domains & Sample quotes from the clinical reports \\
\hline Telehealth and apps availability & $\begin{array}{l}\text { "We are still doing some telehealth and telephone visit as needed." } \\
\text { "Patients are offered telehealth appts \& medication refill appts are also being offered via telehealth and } \\
\text { telephone." } \\
\text { "We have virtual visit capabilities for all patients with regular follow-up appointments if patients have } \\
\text { computer and internet access at home. For Ryan White patients, MCMs will bring laptop and hot spot to } \\
\text { the patient's home, get them online with provider and step out of the room.... We are using the Polycom } \\
\text { Bridge system for virtual visits." } \\
\text { "Continuing to utilize telehealth options versus in-person visits where possible." } \\
\text { "Utilizing Healow as much as possible." }\end{array}$ \\
\hline Healthcare provider availability & $\begin{array}{l}\text { "Effective June } 8,2020 \text { : MCMs working from home using remote desktop to access PE, email etc. and } \\
\text { telephone extensions forwarded to their respective cell phones; MCMs are rotating working "in office" } \\
\text { at least two days a week ( } 7 \text { per day) providing services by appointment only. Medical providers practic- } \\
\text { ing telehealth as appropriate and seeing patients face-to-face as necessary. Contract ID provider seeing } \\
\text { patients (face to face) in AD office, while } 2 \text { part-time providers are practicing telemedicine.... Clinical } \\
\text { CM "in office" to assist provider(s) } 4 \text { days/wk." } \\
\text { "MCMs are reaching out to clients who haven't picked up meds and offering medication delivery upon } \\
\text { request. Housing Case Manager \& MCMs continue to provide housing and referral services. Our } \\
\text { Housing facility operating as usual; Behavioral health provider returns to office full-time this week \& } \\
\text { continues to offer services by teleconference and face to face." } \\
\text { "No office appts for MCM, all MCMs working remotely from home, they VPNs obtained from DHEC for } \\
\text { access to PE." } \\
\text { "Effective June 1st more staff will be called back into the office." } \\
\text { "Approximately half of the staff are back in the office.Others are still working from home remotely to } \\
\text { contact our patients for appointments and any immediate needs. " } \\
\text { "Most providers are now back to seeing patients in clinic along with virtual visits." } \\
\text { "The front office will be open on a reduced schedule AH is the Office Manager on Duty. This is to } \\
\text { facilitate Case Management staff and medication pick up and other essential support services for clients. } \\
\text { Decisions going forward will be made daily/weekly as to our re-opening with full staffing. All staff } \\
\text { available via email/phone we are working via remote." }\end{array}$ \\
\hline
\end{tabular}

Note: All the identifiable information (e.g., names or locations) were removed in the quotes to protect the identity of the respondents

system). In some clinics, MCMs were rotating "in office" at least 2 days a week, providing services by appointment only. One clinic reported that their MCMs reached out to clients who had not picked up medicines and offered medication delivery. Since June 2020, more providers and staff have returned to the office. One clinic staff said, "Decisions going forward will be made daily/weekly as to our re-opening with full staffing."

\section{Geospatial Patterns of HIV Service Interruption}

The county-level geospatial pattern of the HIV service interruption among all HIV clinics (except the one that provided state-wide service), along with the geospatial pattern of COVID-19 cases in SC is shown in Fig. 1. Generally, most of the service catchment areas were moderately affected by the COVID-19 outbreak in terms of confirmed COVID19 cases per capita. In the Upstate area no clinic reported "complete interruption" of HIV services. Geospatial heterogeneity in terms of HIV service interruption existed. However, the geospatial pattern of HIV service interruption did not exactly overlap with the density of COVID-19 cases at county level.

\section{Association Between Interruption and Health Disparity Factors}

The results of descriptive analysis and ANOVA analysis are shown in Table 2. Among the service catchment areas of the HIV clinics, the mean rate of PLWH in 2018 and the mean rate of confirmed COVID-19 cases as of June 13, 2020 was 429 and 416 per 100,000 , respectively. The median household income averaged $\$ 43,124(\mathrm{SD}=5367)$. The mean of Gini coefficients was .46. The percentage of uninsured population was $13.5 \%$ on average (statewide: $10.5 \%$ ) [13]. Among these counties, the percentage of people in the county living in poverty was approximately $20 \%$ (statewide: $15.3 \%$ ) [13]. On average, about $83 \%$ of population had graduated from high school. ANOVA analysis did not detect significant difference by HIV service interruption types in terms of SES indicators except the percentage of uninsured population.

The geospatial distribution pattern of PLWH was similar to the distribution pattern of confirmed COVID-19 cases. HIV service interruption pattern did not consistently correspond with the density of COVID-19 cases. While the clinics that reported no interruption in services were in areas with the lowest rate of COVID-19 cases, 
Fig. 1 Geospatial heterogeneity of HIV service interruption and confirmed COVID-19 cases per capita

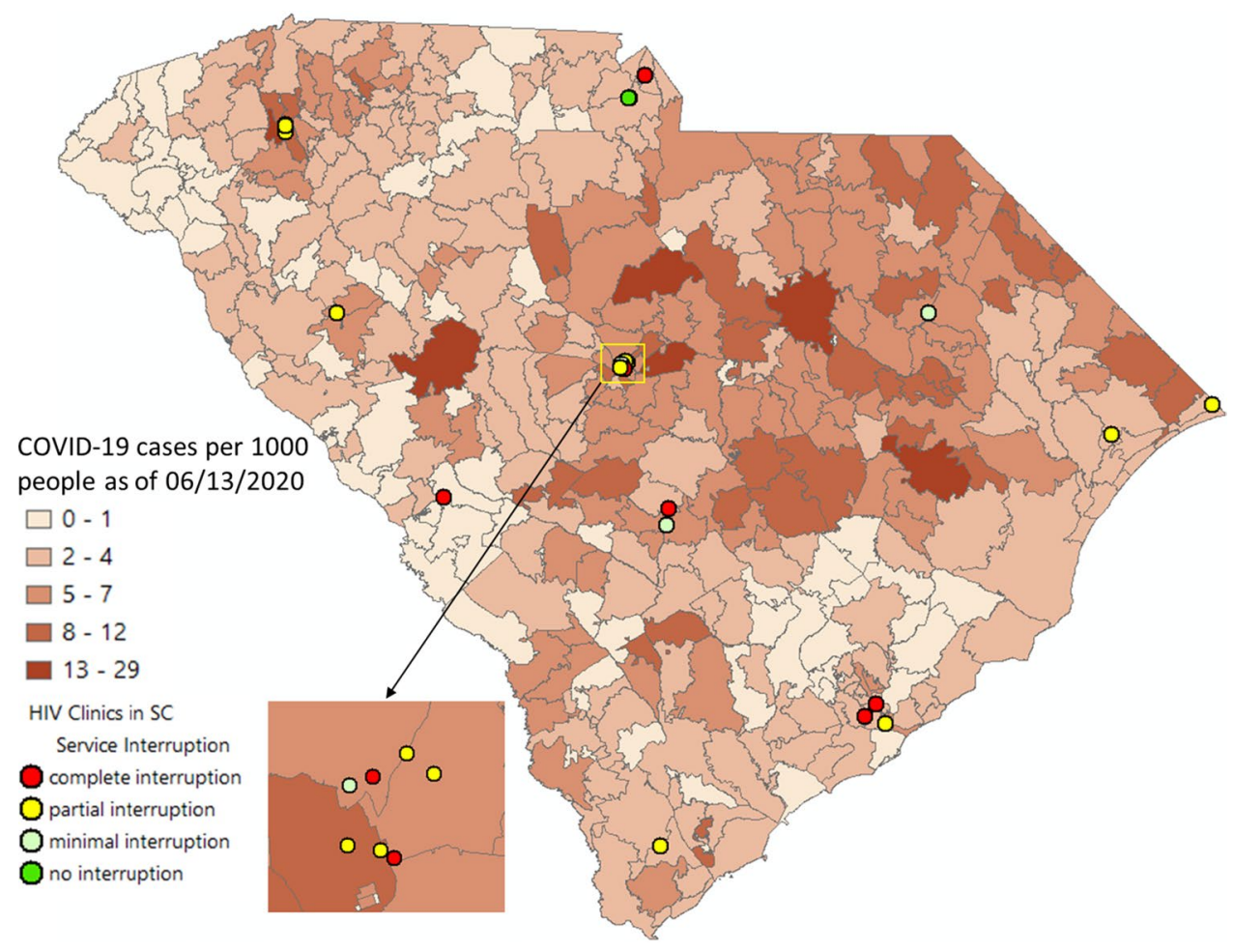

Table 2 HIV and COVID-19 epidemic and socioeconomic characteristics of the service enactment area by HIV service interruption type

\begin{tabular}{|c|c|c|c|c|c|c|c|c|}
\hline \multicolumn{2}{|c|}{ HIV service interruption } & \multirow{2}{*}{$\begin{array}{l}\text { Rates of } \\
\text { PLWH (per } \\
100,000) \\
286.97\end{array}$} & \multirow{2}{*}{$\begin{array}{l}\text { Rates of COVID- } \\
19 \text { cases (per } \\
100,000) \\
270.66\end{array}$} & \multirow{2}{*}{$\begin{array}{l}\text { Percent of } \\
\text { uninsured }\end{array}$} & \multirow{2}{*}{$\begin{array}{l}\begin{array}{l}\text { Median } \\
\text { household } \\
\text { income }\end{array} \\
44,479.70\end{array}$} & \multirow{2}{*}{$\begin{array}{l}\text { Gini coefficient } \\
.46\end{array}$} & \multirow{2}{*}{$\begin{array}{l}\text { Percent of } \\
\text { living in } \\
\text { poverty }\end{array}$} & \multirow{2}{*}{$\begin{array}{l}\text { Percent of high } \\
\text { school graduation }\end{array}$} \\
\hline No interruption & Mean & & & & & & & \\
\hline $\begin{array}{l}\text { Clinic } \#=2 \\
\text { County\# }=8^{*}\end{array}$ & SD & 20.32 & 37.71 & .24 & 2898.15 & .00024 & 1.19 & 1.70 \\
\hline $\begin{array}{l}\text { Minimal interrup- } \\
\text { tion }\end{array}$ & Mean & 552.36 & 545.32 & 13.36 & $37,802.81$ & .47 & 23.61 & 80.51 \\
\hline $\begin{array}{l}\text { Clinic\# }=3 \\
\text { County\# }=17^{*}\end{array}$ & SD & 50.35 & 211.40 & .59 & 4097.45 & .016 & 3.19 & 2.10 \\
\hline Partial interruption & Mean & 415.32 & 432.94 & 14.13 & $43,315.44$ & .46 & 19.45 & 83.51 \\
\hline $\begin{array}{l}\text { Clinic\# }=14 \\
\text { County\# }=68^{*}\end{array}$ & SD & 150.45 & 178.69 & 1.42 & 5059.56 & .012 & 3.19 & 2.87 \\
\hline $\begin{array}{l}\text { Complete inter- } \\
\text { ruption }\end{array}$ & Mean & 444.25 & 364.32 & 12.52 & $44,854.44$ & .45 & 19.46 & 83.83 \\
\hline $\begin{array}{l}\text { Clinic\# }=7 \\
\text { County }=32 *\end{array}$ & SD & 135.68 & 193.15 & .55 & 6521.90 & .016 & 5.14 & 3.39 \\
\hline \multirow[t]{2}{*}{ Total } & Mean & 429.04 & 414.95 & 13.49 & $43,124.08$ & .46 & 19.85 & 83.17 \\
\hline & SD & 141.48 & 183.83 & 1.38 & 5367.23 & .014 & 3.72 & 2.90 \\
\hline
\end{tabular}

Note: * indicates the counties in which the clinics were not mutually exclusive

some clinics that reported minimal interruptions were in areas with the highest rate of COVID-19 cases. The percentage of uninsured population was significantly different by HIV service interruption type $(\mathrm{F}=3.987, \mathrm{P}=.02)$, indicating a potential correlation between health insurance coverage and the HIV service interruption. Post-hoc pairwise comparison (data not shown) suggested that the service catchment areas with no interruption in services had a lower percentage of uninsured than those areas with minimal service interruption, partial service interruption, and 
complete service interruption, although not all of these differences reached statistical significance.

\section{Discussion}

Using mixed-method data collected from 27 HIV clinics in SC, the current study examined HIV service interruptions in SC during the COVID-19 outbreak. We demonstrated geospatial pattern of the HIV service interruption, and explored the potential socioeconomic correlates with the service interruption. Of our sample, nearly $82 \%$ of the HIV clinics were either partially interrupted $(56 \%)$ or completely closed (26\%) during the COVID-19 outbreak. The COVID-19 disrupted regular operation routine, reduced office hours, and decreased the availability of healthcare providers and staff in HIV clinics. Although most of the clinics still provided core HIV services (e.g., medicine refilling, HIV testing), they had to suspend or cancel faceto-face counseling and social support group. Geospatial heterogeneity in terms of HIV service interruption existed. However, the geospatial pattern of HIV service interruption did not exactly overlap with the density of confirmed COVID-19 cases in the service catchment area. The areas with a higher insurance coverage (i.e., a lower percentage of uninsured population) tended to show less HIV service interruption.

Telehealth and mobile applications played an important role in retaining HIV services and delivering other supportive services during the COVID-19 outbreak. Our findings provide further empirical evidence for the value and need of telehealth as a convenient and inexpensive care option during disasters and public health crisis [22-24]. However, our qualitative study suggested that not all HIV clinics were able to make a timely shift to a telehealth system. Four of the HIV clinics did not have capacity to support virtual visit for patients. In addition, many patients who did not have computer or internet access at home might not be able to benefit from telehealth, leading to a widening disparity in care. Further implementation science studies are needed to explore the facilitators and barriers for scaling-up telehealth in responding to COVID-19 pandemic and other public health emergencies.

The geospatial distribution of PLWH largely overlapped with the geospatial distribution of confirmed COVID-19 cases through June 2020. This result suggests the importance to integrate the efforts of responding dual epidemics of HIV and COVID-19. Some HIV clinics reported that they provided COVID-19 testing for their communities in partnership with SC DHEC. These practices are consistent with the recent recommendation in the literature to take advantage of COVID-19 contact tracing as a great opportunity for a more coverage of HIV testing [25]. Future studies on needs assessment and infrastructure evaluation are warranted to explore the feasibility and acceptability of providing integrated COVID-19 and HIV testing and services for local communities based on existing HIV testing program or infrastructures available at HIV clinics.

Our findings suggested a big variation across SC in extent of HIV service interruption with a range from no interruption to complete interruption of services. Most of HIV clinics had adapted flexible strategies to deal with the crisis caused by COVID-19, but they still showed difference in operational hours, healthcare provider availability, and scope of HIV services during the outbreak. This gap in responding to the public health crisis could not be fully explained by the COVID-19 itself. Existing socioeconomic disparities at county level might contribute to the gap in health service delivering. For example, the percentage of uninsured population in service catchment area was related to the service interruption of HIV clinics. We may need to further examine the characteristics of HIV clinics in terms of their financial situation, human resources, and infrastructure of telehealth to explore if and how existing variation in health infrastructure is associated with disparity of HIV service interruption.

The disparity of HIV service interruption during a public health crisis such as COVID-19 is an important issue for the healthcare systems and policy makers to consider as such interruption may further amplify the disparities in HIV treatment cascade in settings with a significant HIV burden or among various vulnerable populations. In the global context, there might be 500,000 additional deaths due to a 6-month interruption of ART in Africa [26]. In the United States, a Boston health center reported that disruptions in Pre-Exposure Prophylaxis (PrEP) care were more prominent among vulnerable population [27]. In Maryland, a comprehensive patient and family centered HIV primary care was interrupted among racial, sexual and gender minority youth living with HIV, which might impede their medicine adherence and clinical improvement [28]. To prevent the further widening of the disparities in existing HIV care system, strengthening and empowering community partners of HIV clinics could be essential for more effective and accountable responses to public health emergencies [29-31].

The current study is subject to several limitations. First, qualitative data was collected within 2 months and reports of the HIV clinics were obtained in different dates. Giving the rapidly evolving COVID-19 pandemic, the information we collected might lag behind the changes made by the clinics in response to the pandemic. Second, we were not able to quantify the HIV service interruption based on existing qualitative information. Although we have identified five domains to assess service interruptions, lack of complete and systematic assessment prevented us from developing a numeric index to measure HIV service interruption. The categorization of HIV clinics by a medical expert and other 
team member might introduce subjective bias. Third, we only included the Ryan White HIV clinics in the analysis because of the availability of program reports. Given the large state-wide coverage of Ryan White programs in providing HIV service, the findings may serve as a good presentation of the HIV service interruption issues in SC. Fourth, the service catchment areas of the HIV clinics were not mutually exclusive. Thus, one county might be included in the calculation of factor variables for more than one HIV clinics. However, since the analysis was based on clinic level, we do not believe that the inclusion of same county level data for different clinics would pose a serious threat to the internal validity of the clinic-level analysis. Finally, the small sample size of the quantitative study limited the power to detect statistically significant correlates.

Despite these limitations, the current study is one of the first efforts to examine the disparity of HIV service interruption and identified the socioeconomic correlates of this disparity. Our findings show that the COVID-19 pandemic added additional burdens to HIV care system in SC. A rapid shift to telehealth system and integration of HIV and COVID-19 care might be effective strategies to respond to this public health crisis. However, existing socioeconomic disparity might contribute to the gap in HIV service interruption, leaving PLWH in low SES communities more vulnerable. We call for more attentions to this disparity in HIV service delivery in both clinics' capacity building and the federal or state support to HIV service programs in fighting the COVID-19 pandemic.

Acknowledgements Research reported in this publication was supported by the National Institute of Allergy and Infectious Diseases of the National Institutes of Health under Award Number R01AI1272034S1 and the University of South Carolina Office of Vice President for Research COVID-19 grant (USCIP 80003673). The content is solely the responsibility of the authors and does not necessarily represent the official views of the National Institutes of Health and University of South Carolina.

\section{References}

1. World Health Organization. Coronavirus disease (COVID-19) Pandemic 2020. https://www.who.int/emergencies/diseases/novel -coronavirus-2019.

2. World Health Organization. WHO Director-General's opening remarks at the media briefing on COVID-19-11 March 2020. 2020. March 22.

3. World Health Organization. Coronavirus disease (COVID-19) Situation Dashboard. 2020. June 21.

4. Centers for Disease Control and Prevention. People of any age with underlying medical conditions 2020 [updated June 25, 2020]. https://www.cdc.gov/coronavirus/2019-ncov/need-extra-preca utions/people-with-medical-conditions.html?CDC_AA_refVa $1=$ https $\% 3 \mathrm{~A} \% 2 \mathrm{~F} \% 2 \mathrm{Fwww} . c d c$. gov $\% 2$ Fcoronavirus $\% 2 \mathrm{~F} 201$ 9-ncov\%2Fneed-extra-precautions\%2Fgroups-at-higher-risk.html.
5. Centers for Disease Control and Prevention. COVID-19: What people with HIV should know 2020. https://www.cdc.gov/coron avirus/2019-ncov/specific-groups/hiv.html.

6. National Institute of Health. Interim Guidance for COVID-19 and Persons with HIV 2020. https://aidsinfo.nih.gov/guidelines $/ \mathrm{html} / 8 /$ covid-19-and-persons-with-hiv--interim-guidance-/0.

7. Beima-Sofie K, Ortblad KF, Swanson F, Graham SM, Stekler JD, Simoni JM. "Keep It Going if You Can": HIV Service Provision for priority populations during the COVID-19 pandemic in Seattle, WA. AIDS Behav. 2020. https://doi.org/10.1007/ s10461-020-02902-5.

8. Ridgway JP, Schmitt J, Friedman E, Taylor M, Devlin S, McNulty M, et al. HIV care continuum and COVID-19 outcomes among people living with HIV during the COVID19 pandemic, Chicago, IL. AIDS Behav. 2020. https://doi. org/10.1007/s10461-020-02905-2.

9. Zhang CH, Schwartz GG. Spatial disparities in coronavirus incidence and mortality in the United States: an ecological analysis as of May 2020. J Rural Health. 2020;36:433-45.

10. South Carolina Department of Health and Environment Control. SC testing data \& projections (COVID-19). 2020.

11. GoLaurens.com. COVID-19 reported in all 46 counties across SC, 4 cases in Laurens County. April 2, 2020. https://www. newsbreak.com/south-carolina/columbia/news/0OdiqYtQ/covid -19-reported-in-all-46-counties-across-sc-4-cases-in-laurenscounty.

12. US Department of Health Human Services. Ending the HIV epidemic: a plan for America. 2019.

13. South Carolina Department of Health and Environment Control. An epidemiologic profile of HIV and AIDS in South Carolina 2019. https://www.scdhec.gov/sites/default/files/media/docum ent/2019-Epi-Profile.pdf.

14. South Carolina Department of Health and Environment Control. SC HIV Service Providers: Ryan White 2018. Part B. https://scdhe c.gov/sites/default/files/media/document/STD\%20HIV\%20Par t\%20B\%20Service\%20Providers\%2011092018.pdf. Part C. https ://www.scdhec.gov/sites/default/files/docs/Health/docs/stdhiv/ STD\%20HIV\%20Part\%20C\%20Service\%20Providers.pdf.

15. Health Resources and Services Administration. About Ryan White HIV/AIDS Program 2019 [updated February 2019]. https://hab. hrsa.gov/about-ryan-white-hivaids-program/about-ryan-white -hivaids-program.

16. Health Resources and Services Adminstration. What are the demographic characteristics of Ryan White HIV/AIDS Program clients in South Carolina? 2018. https://hab.hrsa.gov/stateprofi les2018/\#/profile.

17. United States Census Bureau. Small area income and poverty estimates. Table 1: 2916 poverty adn median income estimates: counties. 2016.

18. United States Census Bureau. American community survey 5-year estimates, 2016. Table DP02: educational attainment for the population 25 years and over: counties. 2016.

19. United States Census Bureau. American community survey 5-year estimates, 2016. Table B19083: Gini Index of income inequality. 2016.

20. United States Census Bureau. Small area health insurance estimates, 2016: Health insurance coverage status by age, race, hispanic origin, sex and income for counties and states. 2016.

21. Strauss A, Corbin J. Grounded theory methodology. Handb Qual Res. 1994;17(1):273-85.

22. Duffy S, Lee TH. In-person health care as option B. N Engl J Med. 2018;378(2):104-6.

23. Hollander JE, Carr BG. Virtually perfect? Telemedicine for COVID-19. N Engl J Med. 2020;382(18):1679-81.

24. Lurie N, Carr BG. The role of telehealth in the medical response to disasters. JAMA Intern Med. 2018;178(6):745-6. 
25. Nosyk B, Armstrong WS, Del Rio C. Contact tracing for COVID19: an opportunity to reduce health disparities and end the HIV/ AIDS Epidemic in the US. Clin Infect Dis. 2020. https://doi. org/10.1093/cid/ciaa501.

26. Hogan AB, Jewell B, Sherrard-Smith E, Vesga J, Watson OJ, Whittaker C, et al. The potential impact of the COVID-19 epidemic on HIV, TB and Malaria in low-and middle-income countries. Imp Coll Lond (01-05-2020). 2020. https://doi.org/10.25561 178670.

27. Adadi P, Kanwugu ON. Living with HIV in the time of COVID19: a glimpse of hope. J Med Virol. 2020. https://doi.org/10.1002/ jmv.26118.

28. Armbruster M, Fields EL, Campbell N, Griffith DC, Kouoh AM, Knott-Grasso MA, et al. Addressing health inequities exacerbated by COVID-19 among youth with HIV: expanding our toolkit. J Adolesc Health. 2020. https://doi.org/10.1016/j.jadoh ealth.2020.05.021.
29. Pinto RM, Park S. COVID-19 Pandemic disrupts HIV continuum of care and prevention: implications for research and practice concerning community-based organizations and frontline providers. AIDS Behav. 2020;24:2486-9.

30. Operario D, King EJ, Gamarel KE. Prioritizing community partners and community HIV workers in the COVID-19 pandemic. AIDS Behav. 2020. https://doi.org/10.1007/s10461-020-02896-0.

31. International AIDS Society. The COVID-19 and HIV: a tale of two pandemics. 2020. https://covid19andhivreport.iasociety.org/.

Publisher's Note Springer Nature remains neutral with regard to jurisdictional claims in published maps and institutional affiliations. 